Francis R. Verdun

Nicolas Theumann

Pierre-Alexandre Poletti

Daniel Gutierrez

Abbas Aroua

Pierre Schnyder

Jean-François Valley

Salah D. Qanadli

\section{Impact of the introduction of 16-row MDCT on image quality and patient dose: phantom study and multi-centre survey}

Received: 4 January 2006

Revised: 1 July 2006

Accepted: 7 July 2006

Published online: 29 August 2006

(C) Springer-Verlag 2006

F. R. Verdun $(\bowtie) \cdot$ D. Gutierrez

A. Aroua $\cdot$ J.-F. Valley

University Institute for Radiation

Physics (IRA-DUMSC),

Grand-Pré 1,

CH-1007 Lausanne, Switzerland e-mail: Francis.Verdun@chuv.ch

Tel.: +41-21-6233434

Fax: +41-21-6233435

N. Theumann · P. Schnyder .

S. D. Qanadli

Department of Diagnostic and Interventional Radiology,

University Hospital of Lausanne

(CHUV), Cardiovascular and

Metabolic Diseases Centre,

1011 Lausanne, Switzerland

P.-A. Poletti

Department of Radiology,

University Hospital of Geneva (HUG),

1211 Genève-14, Switzerland

\begin{abstract}
The purpose was to compare the image quality and patient dose between 4- and 16-row MDCT units and to evaluate the dispersion of the dose delivered for common clinical examinations. Four 4- and 16-row MDCT units were used in the study. Image noise levels from images of a CatPhan phantom were compared for all units using a given $\mathrm{CTDI}_{\mathrm{vol}}$ of $15.0 \pm 1.0 \mathrm{mGy}$. Standard acquisition protocols from ten centres, shifted from 4- to 16-row MDCT (plus one additional centre for 16-row MDCT), were compared for cerebral angiography and standard chest and abdomen examinations. In addition, the protocols used with 16-row MDCT units for diagnosis of the unstable shoulder and for cardiac examinations were also compared. The introduction of 16-MSCT units did not reduce the performance of the detectors.

Concerning the acquisition protocols, a wide range in practice was observed
\end{abstract}

for standard examinations; DLP varied from 800 to $5,120 \mathrm{mGy} . \mathrm{cm}$, 130 to 860 mGy.cm, 410 to $1,790 \mathrm{mGy} . \mathrm{cm}$ and 850 to $2,500 \mathrm{mGy}$. $\mathrm{cm}$ for cerebral angiography, standard chest, standard abdomen and heart examinations, respectively.The introduction of 16-row MDCT did not, on average, increase the patient dose for standard chest and abdominal examinations. However, a significant dose increase has been observed for cerebral angiography. There is a wide dispersion in the doses delivered, especially for cardiac imaging.

Keywords Multi-slice spiral CT . CT dose survey .

Dose reference levels (DRL)

\section{Introduction}

After the introduction of single-slice helical CT (SSCT) in clinical practice in 1989 [1], the next major advance in CT technology was the development of multi-detector helical CT (MDCT) systems. In 1998, several manufacturers launched CT systems capable of acquiring four slices simultaneously, resulting in an improvement in scanner performance that could be used either to scan a larger region of interest (ROI) or to scan an ROI in a shorter time or with an improved spatial resolution compared with SSCT technology [2-5]. These opportunities increased the clinical efficacy of $\mathrm{CT}$ procedures and offered promising new applications in diagnostic imaging [6,7], especially in vascular and cardiac imaging [8-10]. However, despite these promising advances, clinical challenges and limitations remained for four-row MDCT systems. In particular, the spatial resolution of four-row MDCT was limited (nonisotropic resolution) and the scan times for large volumes were sufficiently long that image quality could be impaired, especially for CT angiography applications [11]. The introduction of 16-row systems in 2002 removed these limitations and made possible the routine acquisition of substantial anatomic volumes with isotropic submillimetre spatial resolution and scan times of less than $10 \mathrm{~s}$ for $300 \mathrm{~mm}$ of coverage $[12,13]$. 
The flip side of the expanding use of MDCT systems in clinical practice has been a considerable increase in both the frequency of CT procedures and patient radiation exposure levels. Data from various national surveys have confirmed, as a general pattern, the growing impact of $\mathrm{CT}$ as a major source of patient and population exposure $[14,15]$.

In the UK, the contribution from CT to the collective dose from medical X-rays has more than doubled in the last 10 years to about $47 \%$, in spite of the fact that the total number of CTs performed represents only $9 \%$ of total diagnostic X-ray examinations [16, 17]. In Switzerland, a recent study showed that the introduction of four-row MDCT led to a two-fold increase in the contribution from CT to the collective dose from medical X-rays [18].

The objective of this study is to compare, for three common examinations, the impact of changing from a 4- to a 16-row MDCT unit on image quality and patient dose. Furthermore, protocols used for cardiac examinations with 16-row MDCT units were collected to assess the dose levels associated with this examination.

\section{Materials and methods}

\section{Study design}

This study is in two parts. The first part is devoted to the assessment of changes in image noise behaviour at a given dose for the MDCT unit using 4- and 16-row detector systems. For each manufacturer, two scanners (one 4- and one 16-row system) were characterised using the method described later in this section. The second part of the study consists of two separate surveys: one organised in 2002 concerning the 4-row systems and involving ten centres (major hospitals throughout Switzerland) and a second one organised in 2004 and involving the same centres as well as one additional centre using 16-row systems.

\section{Phantom study}

The following 4- and 16-row MDCT units were included in the study: the 4-row systems: GE LightSpeed QX/i (GE Medical Systems, Milwaukee, Wis.), Marconi $\mathrm{M} \times 8000$ (Philips Medical Systems, Best, The Netherlands), Toshiba Aquilion (Toshiba Medical Systems Europe, Zoetermeer, The Netherlands), and Siemens Volume Zoom (Siemens Medical Systems, Erlangen Germany); 16-row systems: GE LightSpeed Ultrafast 16 , Phillips $\mathrm{M} \times 8000$ IDT 16 , Toshiba Aquilion 16 and Siemens Sensation 16.

Eight units (one 4- and one 16-row from each manufacturer) were selected to investigate the relationship between dose and image noise. All the units displayed the weighted CT dose index $\mathrm{CTDI}_{\mathrm{w}}$ (or $\mathrm{CTDI}_{\mathrm{vol}}$ ) and the dose length product (DLP) corresponding to a standard acquisition protocol. The $\mathrm{CTDI}_{\mathrm{w}}$ data were verified by mea- suring the ${ }_{\mathrm{n}} \mathrm{CTDI}_{\mathrm{w}}$ using a 32-cm diameter CTDI test object and a 10-cm-long CT pencil ionization chamber connected to an electrometer (model 1035-10.3 CTDI chamber and a MDH model 1015 electrometer, Radcal, Monrovia, Calif.), calibrated in RQR9 and RQA9 beams according to IEC 61267 [19] and traceable to the Swiss Federal Office of Metrology.

Images of a commercially available $\mathrm{CT}$ test object Catphan 500 (The Phantom Laboratory, Cambridge, NY), using the additional annulus provided by the manufacturer to simulate the absorption of a standard abdomen (giving a total test object diameter of $30 \mathrm{~cm}$ ), were acquired at $120 \mathrm{kV}$ using a $360^{\circ}$ gantry rotation time of $0.5 \mathrm{~s}$ in the helical mode with a pitch value as close as possible to 1.0 for each unit, and the smallest detector cells available (for example: $16 \times 0.625 \mathrm{~mm}$ for the GE systems or $16 \times 0.75$ for the Siemens systems). All the acquisitions were performed five times successively setting the tube current value in such a way as to obtain a $\mathrm{CTDI}_{\mathrm{vol}}$ of $15.0 \pm 1.0 \mathrm{mGy}$. A 5.0-mm reconstructed slice thickness was then selected for all units using the reconstruction kernel provided by the manufacturer for an abdominal acquisition. Standard deviations (SD) of pixel values were measured in a homogeneous area of about 1,500 pixels in the centre of the field of view (FOV) on five images per unit. A mean value was calculated. The relative variation in standard deviation, $\Delta \mathrm{SD}$, between 4- and 16-row units from the same manufacturer was then calculated according to the following formula:

$$
\Delta S D(\%)=\frac{S D_{16-\text { row }}-S D_{4-\text { row }}}{\left(S D_{16-\text { row }}+S D_{4-\text { row }}\right) / 2} \cdot 100
$$

In order to avoid the comparison of SD values measured on images that differ too much in terms of spatial frequency noise content, noise power spectra were calculated and compared for the 4- and 16-row MDCT of each manufacturer. In each image a homogeneous area of $128 \times 128$ pixels was selected. This part of the image was divided into four image subsets of $64 \times 64$ pixels. A noise power spectrum was then calculated for each of these image subsets [20]. Since the goal of the paper was not the comparison of the performances of each individual unit but the evaluation of the evolution of the technology, intermanufacturer comparisons have not been made.

It is of note that image quality not only depends on image noise, but also on in-plane and longitudinal resolutions. Nevertheless, for each unit a comparable reconstruction field of view and a comparable reconstructed slice thickness were chosen. In such conditions, it appears that image noise is the most critical parameter to be investigated. Since image dose is controlled by the dose received by the detectors, it will strongly depend on the $\mathrm{CTDI}_{\mathrm{vol}}$ parameter. 


\section{Clinical survey}

Ten hospitals (medium-size and university hospitals) that had switched from 4- to 16-row units (keeping the same CT manufacturer) between 2002 and 2004 agreed in 2002 and 2004 to participate in a survey concerning the acquisition protocols for standard examinations. One additional centre participated in only the 16-row unit survey (centre J). Each centre received a form where CT parameters had to be filled out for a target population defined as a patient with a weight of $75 \pm 5 \mathrm{~kg}$ for three clinical indications: (1) suspicion of aneurysm (cerebral CT angiography), (2) pulmonary nodule assessment (CT of the chest) and (3) acute abdominal pain (CT of the abdomen). The CT parameters included tube voltage, tube current and tube rotation time, pitch value, scanned length, number of sequences (unenhanced series; contrast media enhanced series), reconstructed slice thickness and the use of tube current modulation (CT-AEC: CT automatic exposure control device and ECG modulation).

For the 2004 survey dealing with the 16-row systems, four indications were added:

- CT arthrography of the shoulder for unstable shoulder

- Heart:

- Calcium scoring

- Coronary angiography

- With a moderate spatial resolution (reconstruction of millimetre slice thickness)

- With a high spatial resolution (reconstruction of submillimetre slice thickness)

The participants were also asked to record the $\mathrm{CTDI}_{\text {vol }}$ and DLP indicated by the unit after having scanned a standard patient for each of these indications. The results of the survey (anonymously) were then sent to each participant for comments and corrections where justified. All participants gave their informed consent to the study.

\section{Results}

Dose and noise characterization

The results of the normalized $\mathrm{CTDI}_{\mathrm{w}}\left({ }_{\mathrm{n}} \mathrm{CTDI}_{\mathrm{w}}\right)$ measured at $120 \mathrm{kV}$ in the $32-\mathrm{cm}$ CTDI test object are summarized in Table 1. There is good agreement between the values provided by the manufacturer and the measured values, with differences of less than $10 \%$. The normalized $\mathrm{CTDI}_{\mathrm{w}}$ indicated by the unit and assessed in the 16-cm CTDI test object were also verified and agreed with data available in the literature [21]. The comparison of the Wiener spectra for a given manufacturer for the 4- and 16-row units showed no significant difference. An example is shown in Fig. 1. Thus, it is possible to compare the evolution of the
Table 1 Normalised $\mathrm{CTDI}_{\mathrm{w}}$ for the four units involved in the study

\begin{tabular}{llll}
\hline Unit & $\begin{array}{l}\text { Measured }{ }_{\mathrm{n}} \mathrm{CTDI}_{\mathrm{w}} \\
\text { indicated } \\
\text { (mGy/ } \\
\text { bys })\end{array}$ & $\begin{array}{l}\text { Relative difference } \\
\text { between } \\
(\mathrm{mGy} / \mathrm{mAs})\end{array}$ & $\begin{array}{l}\text { measured and } \\
\text { indicated } \\
{ }_{\mathrm{n}} \mathrm{CTDI}_{\mathrm{w}}(\%)\end{array}$ \\
\hline $\begin{array}{l}\text { 4-row LightSpeed } \\
\text { QX/i1 }\end{array}$ & 0.096 & 0.090 & 6.3 \\
$\begin{array}{l}\text { 16-row LightSpeed } \\
\text { Ultrafast1 }\end{array}$ & 0.092 & 0.087 & 5.4 \\
$\begin{array}{l}\text { 4-row Marconi Mx } \\
\text { 80002 }\end{array}$ & 0.073 & 0.068 & 6.8 \\
$\begin{array}{l}\text { 16-row Philips Mx } \\
\text { 8000 IDT2 }\end{array}$ & 0.075 & 0.071 & 5.3 \\
4-row Aquilion3 & 0.116 & 0.105 & 9.2 \\
16-row Aquilion3 & 0.123 & 0.120 & 2.5 \\
4-row Volume Zoom4 & 0.083 & 0.086 & 3.6 \\
16-row Sensation4 & 0.080 & 0.083 & 3.8 \\
\hline
\end{tabular}

noise level for a given dose level between the units of each manufacturer by comparing the standard deviation parameter. The relative variations in standard deviation for a $\mathrm{CTDI}_{\mathrm{vol}}$ of $15.0 \mathrm{mGy}$ when dealing with 4- and 16-row units are summarized in Table 2. No major noise variation was observed for the Toshiba system, whereas noise was significantly reduced for the other systems, as shown as for example in Fig. 2 for the GE units. Thus, for a given $\mathrm{CTDI}_{\mathrm{vol}}$, the use of more rows to acquire the data set not only not led to an increase in image noise, but actually led to an improvement for most of the systems.

The results of the surveys are summarised in Figs. 3, 4, 5, 6 and 7, where the total DLPs for the acquisitions

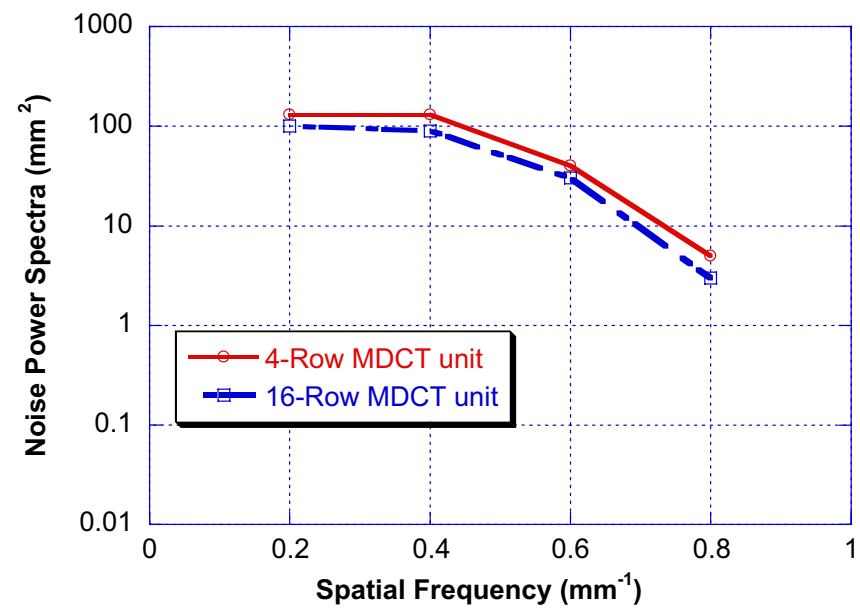

Fig. 1 Comparison of the noise power spectra of the GE 4- and 16row MDCT for a reconstructed slice thickness of $5 \mathrm{~mm}$ and a $\mathrm{CTDI}_{\mathrm{vol}}$ of $15 \mathrm{mGy}$ showing that in the imaging conditions used the spatial frequency content of the noise is comparable for both systems 
Table 2 Relative variation of image noise when switching from a 4to a 16-row unit

\begin{tabular}{lll}
\hline Unit & $\Delta \mathrm{SD}(\%)$ & Centres \\
\hline GE & -35.0 & $\mathrm{~J}, \mathrm{~K}$ \\
Marconi/Philips & -12.0 & $\mathrm{D}, \mathrm{F}$ \\
Toshiba & -2.0 & $\mathrm{~B}, \mathrm{C}, \mathrm{G}$ \\
Siemens & -15.0 & $\mathrm{~A}, \mathrm{E}, \mathrm{H}, \mathrm{I}$ \\
\hline
\end{tabular}

(including all acquisition sequences) are plotted as a function of the averaged (over the different acquisition sequences) $\mathrm{CTDI}_{\mathrm{vol}}$ (representative of the noise content in the reconstructed slices). A "+" or "-" sign has been added to the letter attributed to the centre to indicate whether the DLP has been increased or decreased when switching from a 4- to a 16-row system. In such graphs, if the scan lengths and number of sequences were similar for all protocols, then a linear relationship between the data would be expected since the DLP is the result of the multiplication of the $\mathrm{CTDI}_{\mathrm{vol}}$ by the scanned length. When one uses several acquisition sequences, using the same $\mathrm{CTDI}_{\mathrm{vol}}$, the total DLP of the examination is shifted towards high DLP values since the scanned length has to be added in the calculation. The number of sequences used for each examination is given in Table 3.

The data corresponding to the protocols used for the cerebral angiography for a suspected aneurysm are shown in Fig. 3. The results show a large spread in $\mathrm{CTDI}_{\mathrm{vol}}$ and DLP values. $\mathrm{CTDI}_{\mathrm{vol}}$ values vary from 20 to $100 \mathrm{mGy}$ and DLP varies from 800 to $5,120 \mathrm{mGy} . \mathrm{cm}$, corresponding to an effective dose range of 1.8 to $11.8 \mathrm{mSv}$ with a mean effective dose of $3.1 \mathrm{mSv}$ (from Table 4: average DLP of 2,544 mGy. $\mathrm{cm}$; conversion coefficient of $0.023 \mathrm{mSv} \cdot \mathrm{mGy}^{-1} . \mathrm{cm}^{-1}$ [22]). One centre (centre I) that does not perform neuro-radiological examinations did not send a protocol for this indication. It is of note that the two centres that did not use a reconstruction slice thickness of $1.5 \mathrm{~mm}$ or smaller, but a reconstruction slice thickness of $3.0 \mathrm{~mm}$, did not choose particularly low $\mathrm{CTDI}_{\mathrm{vol}}$ values (centres $\mathrm{E}$ and $\mathrm{F}$ ). As shown in Table 3, only one centre used a single acquisition sequence, whereas the other centres used two to three acquisition sequences.

The results for chest examinations for nodule assessment are presented in Fig. 4. The graph indicates relatively good coherency between the centres concerning the scan lengths and the number of sequences (Table 3 ). In contrast to Fig. 3, where no dose reduction was observed between 4and 16-row MDCT units, four centres have drastically reduced their dose levels after changing units (centres A, D, $\mathrm{E}$ and $\mathrm{H}$ ), four centres have kept their dose levels comparable (centres B, F, I and K) and two centres have drastically increased their dose levels (centres $\mathrm{C}$ and $\mathrm{G}$ ). In centre $\mathrm{G}$, the decrease in $\mathrm{CTDI}_{\mathrm{vol}}$ that was observed was counteracted by the use of three acquisition sequences rather than the single sequence used with the 4-row unit. This led to a very high DLP value. The reconstructed slice thickness used by the centres varied from 2.0 to $5.0 \mathrm{~mm}$ without any significant impact on the $\mathrm{CTDI}_{\mathrm{vol}}$ used. $\mathrm{CTDI}_{\mathrm{vol}}$ values vary from 3.0 to $10.0 \mathrm{mGy}$, whereas DLP varies from 130 to $860 \mathrm{mGy} . \mathrm{cm}$, corresponding to an effective dose range of 2.2 to $14.6 \mathrm{mSv}$ with a mean effective dose of $5.7 \mathrm{mSv}$ (Table 4: average DLP of $337 \mathrm{mGy} . \mathrm{cm}$; conversion coefficient of $0.017 \mathrm{mSv}^{\mathrm{mGy}}{ }^{-1}$. $\left.\mathrm{cm}^{-1}[22]\right)$.

Figure 5 summarises the results for the protocols for abdominal examinations in the case of acute pain. In contrast to Fig. 4, points on the graph are spread over a wide range of $\mathrm{CTDI}_{\mathrm{vol}}$ and DLP values without any noticeable alignment trend. When comparing 4- to 16-row MDCT protocols, significant dose reductions were observed for four centres (centres A, D, E and G), four centres have kept their dose levels comparable (centres B, C, I and $\mathrm{K}$ ), and two centres have drastically increased their dose levels (centres $\mathrm{F}$ and $\mathrm{H}$, which use three and four acquisition sequences, respectively). The reconstructed
Fig. 2 Comparison of image noise content on images obtained on a GE 4- and 16-row MDCT (respectively, a and b) for a reconstructed slice thickness of $5 \mathrm{~mm}$ and a CTDI ${ }_{\mathrm{vol}}$ of $15 \mathrm{mGy}$ showing that in the imaging conditions used, the noise level of the 16-row MDCT unit was significantly lower than the one produced with the 4-row MDCT unit
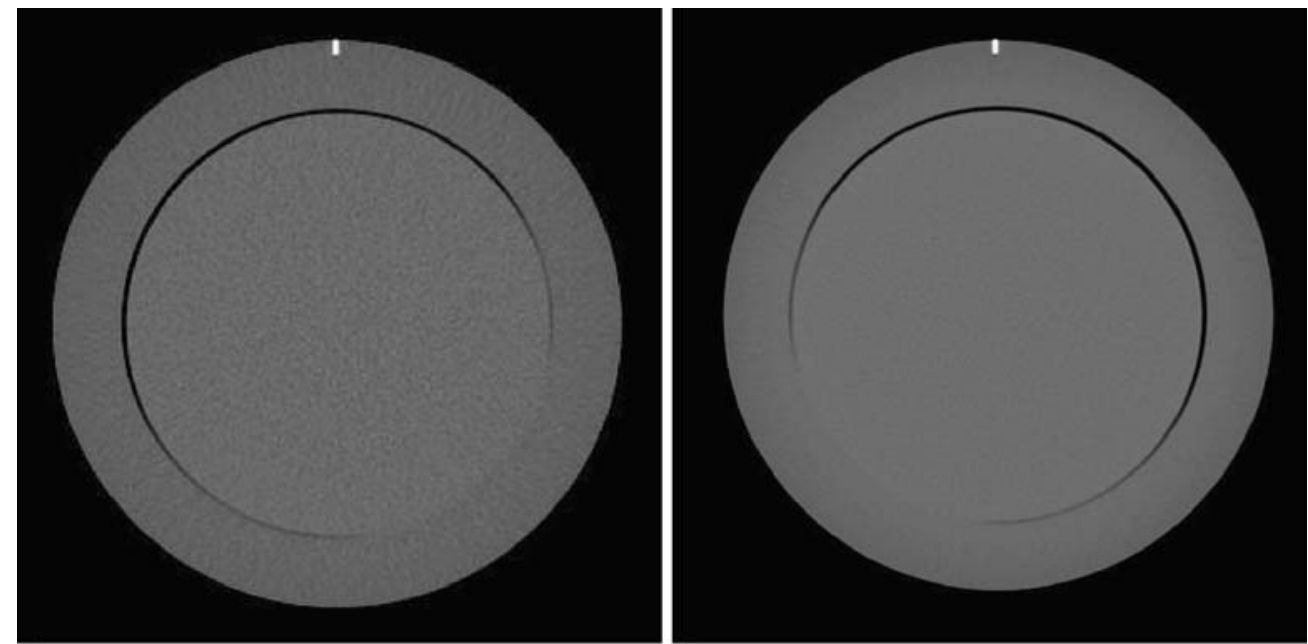

a) b) 
Fig. 3 Comparison of the dosimetric data for 4- and 16-row MDCT protocols for cerebral angiographies in the case of a suspected aneurysm. A "+" or "-" sign has been added to the letter attributed to the centre to indicate whether the DLP was increased or decreased when switching from a 4- to a 16-row system

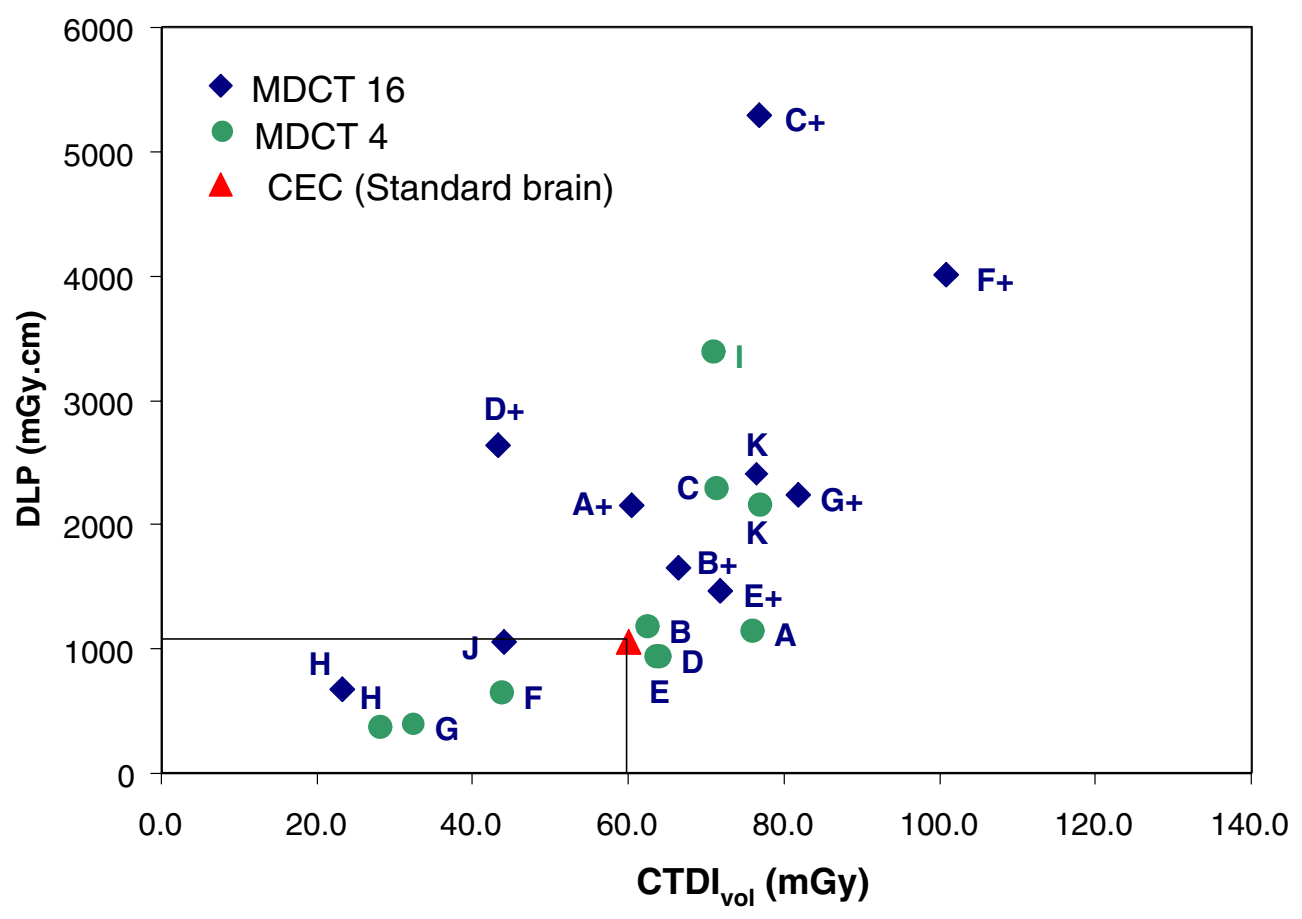

slice thickness employed by the centres varied from 2.0 to $7.0 \mathrm{~mm}$ without any significant impact on the $\mathrm{CTDI}_{\mathrm{vol}}$ used. CTDI ${ }_{\mathrm{vol}}$ values vary from 8.0 to $20.0 \mathrm{mGy}$, and DLP varies from 410.0 to $1,790.0 \mathrm{mGy} . \mathrm{cm}$, corresponding to an effective dose range of 6.2 to $26.9 \mathrm{mSv}$ with a mean effective dose of $12.2 \mathrm{mSv}$ (Table 4; average DLP of
$815 \mathrm{mGy} . \mathrm{cm}$; conversion coefficient of $0.015 \mathrm{mSv} \cdot \mathrm{mGy}^{-1}$. $\left.\mathrm{cm}^{-1}[22]\right)$.

The results presented in Fig. 6 are from the survey of CT arthrography of the shoulder for an examination of an unstable shoulder and do not include data from 4-row MDCT units. The linear trend between the $\mathrm{CTDI}_{\mathrm{vol}}$ and
Fig. 4 Comparison of the dosimetric data for 4- and 16-row MDCT protocols for chest examinations in the case of a suspicious mass. A "+" or "-" sign has been added to the letter attributed to the centre to indicate whether the DLP was increased or decreased when switching from a 4- to a 16-row system

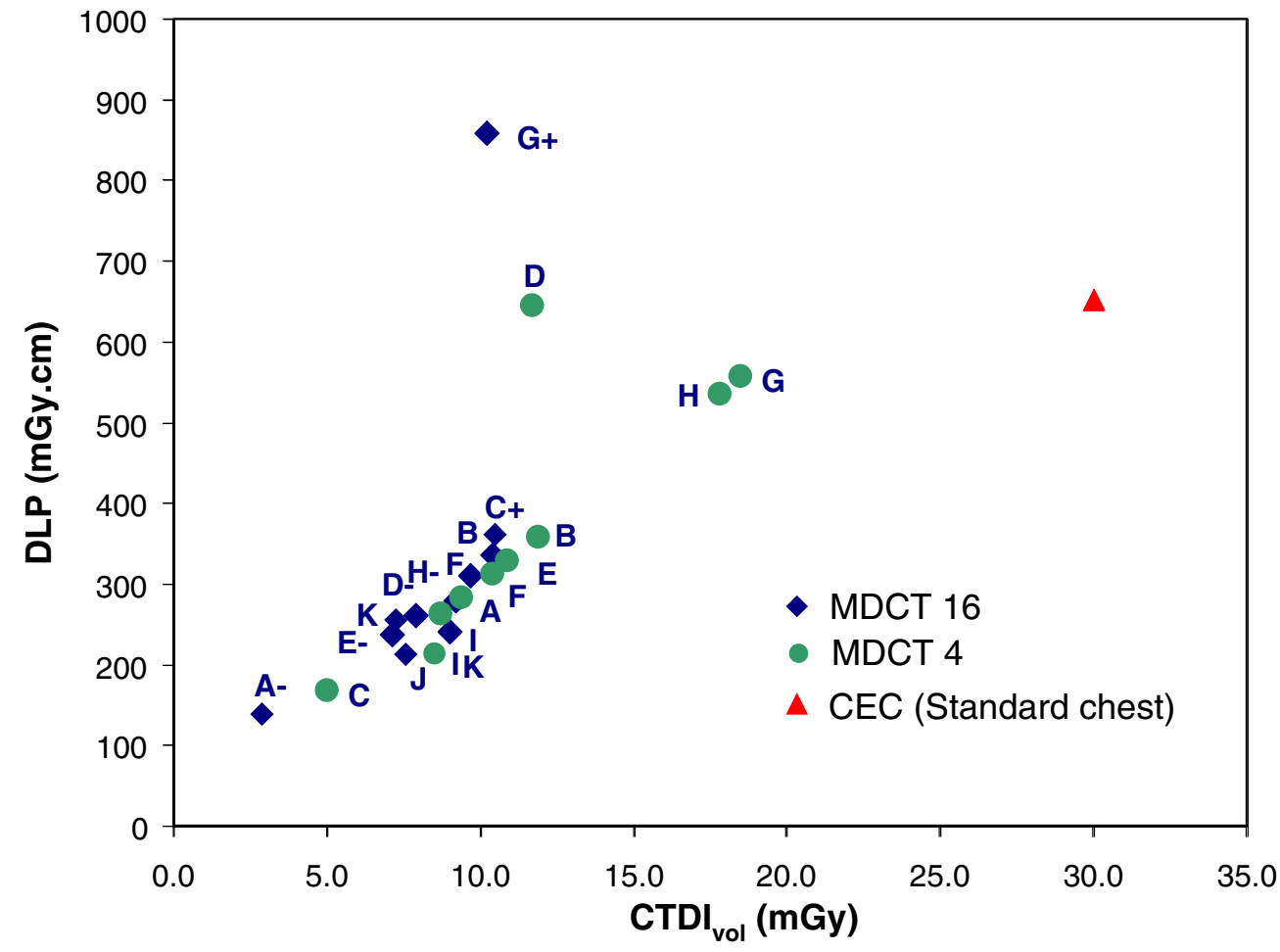


Fig. 5 Comparison of the dosimetric data for 4- and 16-row MDCT protocols for abdominal examinations in the case of abdominal acute pain. A "+" or "-" sign has been added to the letter attributed to the centre to indicate whether the DLP was increased or decreased when switching from a 4- to a 16-row system

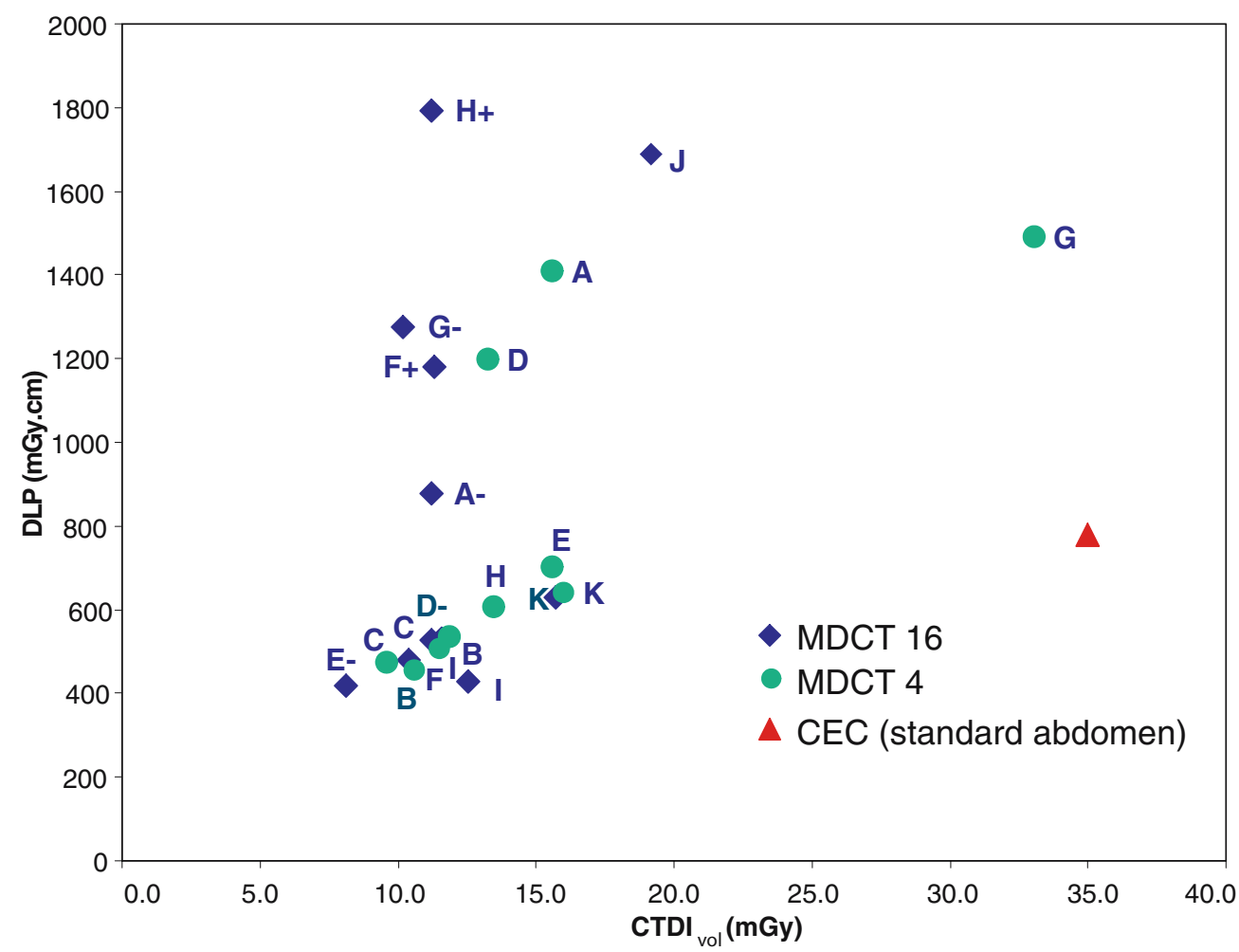

the DLP shows that the centres work with images with significant differences in the image noise level. As for the previous examinations, no correlation could be found between the values of the CTDI $\mathrm{vol}_{\mathrm{vo}}$ and the reconstructed slice thickness (indicated between brackets in Fig. 6). Of the 11 centres surveyed, 3 indicated that this examination was exclusively performed with MRI. The CTDI $_{\mathrm{vol}}$ varies from 11.0 to $119.0 \mathrm{mGy}$ (DLP range of 190.0 to
Fig. 6 Results of the survey for 16-row MDCT only for protocols for the examination of an unstable shoulder. The reconstructed slice thickness used by the centre is indicated in brackets

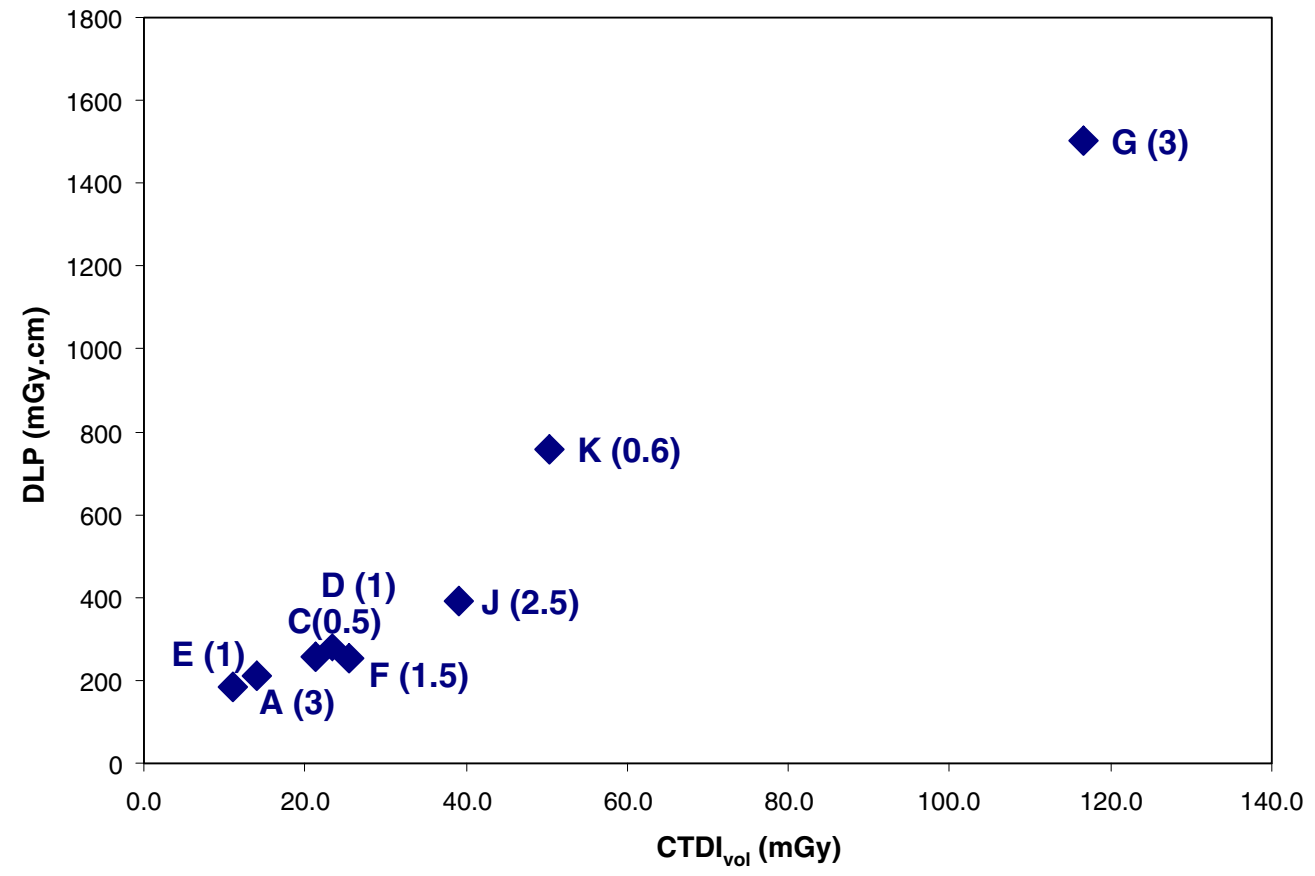


Fig. 7 Results of the survey for 16-row MDCT only for protocols for cardiac examinations. Centres that use tube current modulation are indicated with a star

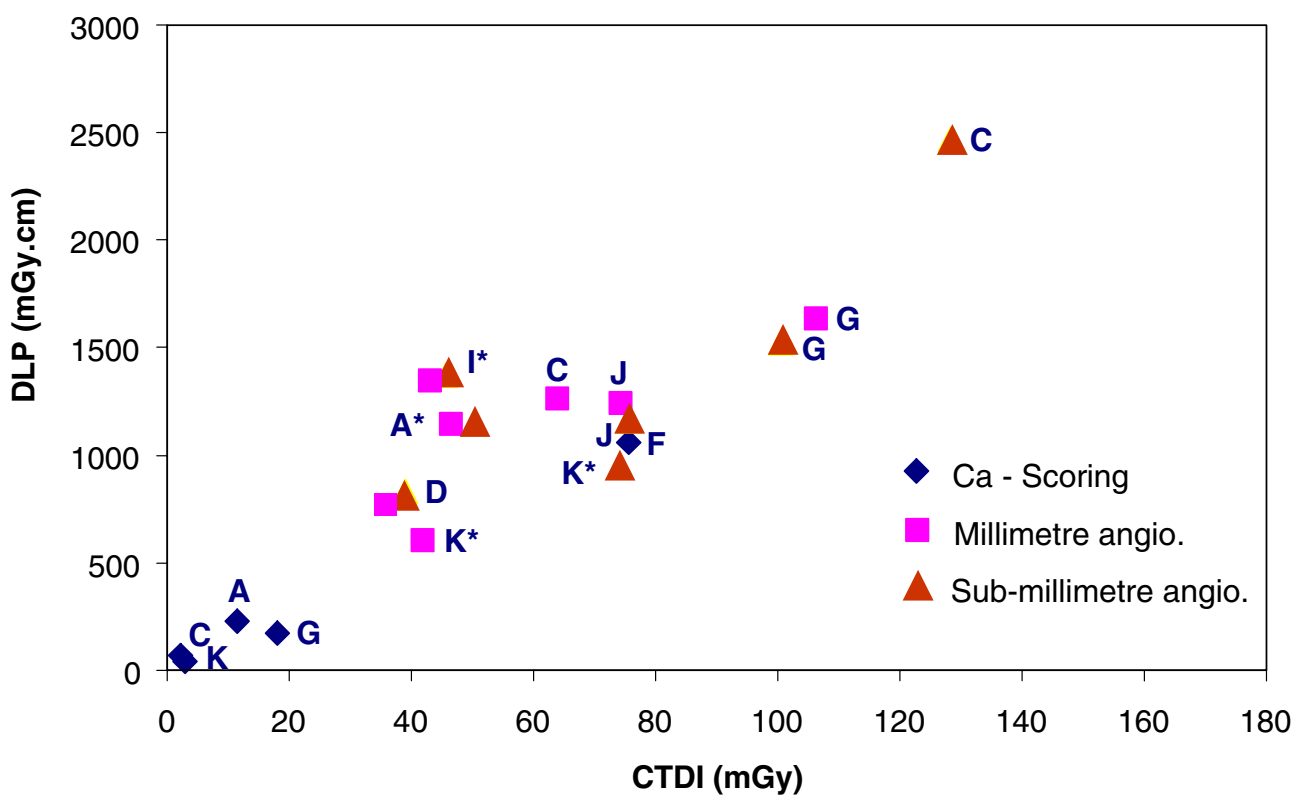

$1,550.0 \mathrm{mGy} . \mathrm{cm})$, resulting in an effective dose range of 3.2 to $26.4 \mathrm{mSv}$ with a mean value of $8.7 \mathrm{mSv}$ (average DLP of 510 mGy.cm; conversion coefficient of $0.017 \mathrm{mSv}$. $\left.\mathrm{mGy}^{-1} \cdot \mathrm{cm}^{-1}[22]\right)$.

Figure 7 presents the results for cardiac examinations. Three centres indicated that they did not perform cardiac examinations with their 16-row MDCT, and so only 8 centres are included in this part of the survey. As expected for calcium scoring, all but one centre (centre F) used a relatively low $\mathrm{CTDI}_{\mathrm{w}}$ value $(2.4$ to $18.0 \mathrm{mGy}$ ), giving a range of DLP from 30.0 to $270.0 \mathrm{mGy} . \mathrm{cm}$ and an effective dose range of 0.5 to $4.6 \mathrm{mSv}$ with a mean value of $1.6 \mathrm{mSv}$ (excluding centre $\mathrm{F}$ and using a conversion coefficient from DLP to an effective dose of $0.017 \mathrm{mSv} \cdot \mathrm{mGy}^{-1} . \mathrm{cm}^{-1}$ proposed for exposures in the chest area [22]). The range of doses for coronary CT angiography is relatively large, and again no direct link could be established between the $\mathrm{CTDI}_{\mathrm{vol}}$ used and the reconstructed slice thickness. For coronary CT angiography, the $\mathrm{CTDI}_{\mathrm{vol}}$ varies from 39.0 to $128.0 \mathrm{mGy}$ (DLP range of 850.0 to $2,500.0 \mathrm{mGy} . \mathrm{cm}$ ), giving an effective dose range of 14.5 to $42.5 \mathrm{mSv}$ with a mean value of $25.8 \mathrm{mSv}$. During this survey, only three centres (centres A, I and $\mathrm{K}$ ) indicated the use of ECG tube current modulation. These centres are marked in Fig. 7 with a star and are the centres that delivered low to moderate doses, $\mathrm{x}, \mathrm{y}$ and $\mathrm{z}$, respectively. The mean effective dose for

Table 3 Number of sequences used for the different protocols investigated

1 sequence 2 sequences 3 sequences 4 sequences

\begin{tabular}{lllll}
\hline Brain vascular & $1 / 9$ & $4 / 9$ & $4 / 9$ & - \\
Chest & $9 / 10$ & $1 / 10$ & - & - \\
Abdomen & $6 / 10$ & $1 / 10$ & $2 / 10$ & $1 / 10$ \\
\hline
\end{tabular}

coronary CT angiography with ECG dose modulation was $19.7 \mathrm{mSv}$.

\section{Discussion}

The averaged CT dose indicators collected in these two surveys show that there was a dose increase for cerebral aneurysm assessment when switching from the 4- to the 16-row MDCT units. Some centres drastically increased their $\mathrm{CTDI}_{\mathrm{vol}}$ as well as their DLP levels, whereas others did not modify their $\mathrm{CTDI}_{\mathrm{vol}}$ values, but drastically increased their DLP values by using more acquisition sequences. It is worth noting that the reference value proposed by the CEC for a standard brain acquisition should not be applied to this particular examination since the diagnostic task is different (vascular assessment). A new set of reference values proposed in the UK for acute stroke [17] has been included in Table 4 and indicates that a $\mathrm{CTDI}_{\mathrm{w}}$ of $100 \mathrm{mGy}$ might be acceptable for investigations of certain parts of the brain. It also proposes a DLP of 930 mGy.cm that is 2.7 times lower than the average DLP value collected in this survey for the 16-row MDCT units. Thus, the protocols used for suspected aneurysm in our centres appear to be relatively different from those used in the case of acute stroke. One of the explanations for such a wide spread in doses was the variation in the number of acquisition sequences used by the different centres. It is clear that, in spite of a precise indication such as 'suspicion of cerebral aneurysm', the protocols collected in the survey were probably subject to different interpretations and performed with different objectives depending on the centre-including screening, diagnosis and pre-therapy assessment or follow-up studies. It is critical that this aspect be addressed before establishing the reference dose 
Table 4 Comparison of averaged dose indicators obtained during the 2002 and 2004 surveys with the new set of reference values* proposed in the UK [17]

\begin{tabular}{lllllll}
\hline & $\begin{array}{l}\mathrm{CTDI}_{\mathrm{vol}} 4 \text {-row } \\
(\mathrm{mGy})\end{array}$ & $\begin{array}{l}\mathrm{CTDI}_{\mathrm{vol}} \text { 16-row } \\
(\mathrm{mGy})\end{array}$ & $\begin{array}{l}\mathrm{CTDI}_{\mathrm{vol}} \text { Ref* } \\
(\mathrm{mGy})\end{array}$ & $\begin{array}{l}\text { DLP 4-row } \\
(\mathrm{mGy} . \mathrm{cm})\end{array}$ & $\begin{array}{l}\text { DLP 16-row } \\
(\mathrm{mGy} . \mathrm{cm})\end{array}$ & $\begin{array}{l}\text { DLP Ref* } \\
(\mathrm{mGy} . \mathrm{cm})\end{array}$ \\
\hline Brain vascular & 59 & 76 & $65^{(1)} / 100^{(2)}$ & 1,336 & 2,544 & 930 \\
Chest & 11 & 8.3 & 13 & 346 & 337 & 580 \\
Abdomen & 15 & 13 & 14 & 782 & 815 & 560 \\
\hline
\end{tabular}

(1) cerebrum; (2) post fossa

level concept in the framework of patient dose optimisation. Thus, a consensual approach is needed to describe precisely the indications for which a protocol will be applied before introducing reference dose levels for such examinations.

The results for chest examinations show an up to threefold difference in the dose between centres. In this survey, only one centre used more than one sequence. The justification for more than one sequence was not clear and led to an effective dose of $15 \mathrm{mSv}$. The $\mathrm{CTDI}_{\mathrm{w}}$ value proposed in 1999 by the CEC (30 mGy) is very high in comparison to the values used in our centres (average value of $8.3 \mathrm{mGy}$ ) and also compared with the value now recommended in the UK [17] (13 mGy). Thus, introducing 16-row MDCT units did not, on average, lead to an increase in patient dose and led to CTDI ${ }_{\mathrm{vol}}$ and DLP values lower than the current recommendations.

There was a wide range of the doses and number of acquisition sequences used for the abdominal examination. Nevertheless, as for the chest examination, there was a slight reduction in the average $\mathrm{CTDI}_{\mathrm{vol}}$ used compared with the value used for the 4-row MDCT units (13 mGy for the 16-row compared with $15 \mathrm{mGy}$ for the 4-row). This value is similar to the one proposed in the UK [17] and is significantly lower than the first CEC recommendations [22]. The spread in DLP values is mainly due to the difference in the number of acquisition sequences used. More than half of the centres used only one sequence, whereas the remaining centres performed two to four acquisition sequences. This might be because the indication 'abdominal acute pain' was too vague or was poorly understood [23, 24]. Here also, the setting of reference dose levels for such examinations requires more information on the indication to avoid the comparison of non-comparable acquisition protocols.

The results obtained for the diagnosis of an unstable shoulder with the 16-row MDCT indicate a wide range in practice, especially concerning the reconstructed slice thickness and $\mathrm{CTDI}_{\mathrm{vol}}$ used. Centre $\mathrm{G}$ is certainly not optimised since a $3.0 \mathrm{~mm}$ reconstructed slice thickness was obtained with the highest $\mathrm{CTDI}_{\mathrm{vol}}$ value, resulting in an effective dose of over $25 \mathrm{mSv}$. For this particular examination, one of the advantages of $\mathrm{CT}$ is the possibility to get thinner slices than MRI $[24,25]$. Thus, the use of a reconstructed slice thickness greater than $2.0 \mathrm{~mm}$ is questionable. However, if slice thicknesses in the range of 2.0 to $3.0 \mathrm{~mm}$ are judged adequate by the centres, then the $\mathrm{CTDI}_{\mathrm{vol}}$ should be adapted, keeping in mind that, for a given $\mathrm{CTDI}_{\mathrm{vol}}$, the greater the slice thickness, the lower the image noise level. Precise recommendations should be made in order to improve the present situation.

Finally, the results obtained for cardiac examinations indicate a lack of consensus concerning the $\mathrm{CTDI}_{\mathrm{vol}}$ to be used at the moment. This situation is particularly critical since some of the protocols used deliver relatively high doses to the patient. The use of tube current modulation allowed three centres to work within a CTDI ${ }_{\mathrm{vol}}$ of $70 \mathrm{mGy}$, a value that is already relatively high when dealing with an exposure of the trunk area of the body. Some other centres delivered similar $\mathrm{CTDI}_{\mathrm{vol}}$ values without the use of tube current modulation. Centres $\mathrm{G}$ and $\mathrm{C}$ use $\mathrm{CTDI}_{\mathrm{vol}}$ values that should be carefully monitored since they lead to a high effective dose to the patient. It is important to highlight the fact that values provided in this survey are higher than those reported in prospective studies for research purposes [26]. Probably the research protocols, due to the associated ethical considerations, lead to more efficient dose control and optimisation than the routine practice.

To conclude, we have shown that the evolution of technology when going from a 4- to a 16-row CT has not led to a major loss of efficiency among the manufacturers. This result can explain why for chest or abdominal examinations the introduction of the 16-row technology has not, on average, led to an increase in the patient dose. However, a consensual approach is needed to define the objectives of CT examinations and the number of acquisition sequences and signal-to-noise ratio that is clinically required [27]. A significant dose increase has been observed in cerebral vascular CT examinations with the 16-row technology. This might be because the image quality provided by 4-row systems was not sufficient to allow a proper diagnosis. However, the variation in $\mathrm{CTDI}_{\mathrm{vol}}$ and DLP that was observed should be restricted by a better definition of the acquisition protocol. Cerebral and cardiac angiography protocols should be carefully monitored, especially with 64-row systems, which open the possibility of new investigation procedures. If the introduction of AEC systems allows better control of patient 
exposure, it appears urgent to improve the way protocols are defined to take into account patient benefit and patient risk [28]. The use of reference dose levels is essential to avoid an unnecessary dose burden to the population $[3,4,9]$.
Acknowledgements The authors gratefully acknowledge financial support from the Prorame Foundation and from the Swiss National Science Foundation (grant no. 3200B0-105951).

\section{References}

1. Kalender WA, Seissler W, Klotz E, Vock P (1990) Spiral volumetric CT with single-breath-hold technique, continuous transport, and scanner rotation. Radiology 176:181-183

2. Klingenbeck-Regn K, Schaller S, Flohr T, Ohnesorge B, Kopp AF, Baum U (1999) Subsecond multi-slice computed tomography: basics and applications. Eur J Radiol 31:110-124

3. Fuchs T, Kachelriess M, Kalender WA (2000) Technical advances in multislice spiral CT. Eur J Radiol 36:69-73

4. Dawson P, Lees WR (2001) Multi-slice technology in computed tomography. Clin Radiol 56:302-309

5. Rydberg J, Buckalter KA, Caldemeyer KS, Phillips MD, Conces DJ, Aisen AM, Persohn SA, Kopecky KK (2000) Multisection CT: scanning techniques and clinical applications. Radiographics 20:1787-1806

6. Berland LL, Smith JK (1998) Multidetector-array CT: once again, technology creates new opportunities. Radiology 209:327-329

7. Kopecky KK, Buckwalter KA, Sokiranski R (1999) Multi-slice CT: spirals past single-slice CT in diagnostic efficacy. Diagn Imaging 21:36-42

8. Knez A, Becker CR, Leber A, Ohnesorge B, Becker A, White C, Haberl R, Reiser MF, Steinbeck G (2001) Usefulness of multislice spiral computed tomography angiography for determination of coronary artery stenoses. Am J Cardiol 88:1191-1194

9. Nieman K, Rensing BJ, van Geuns RJ, Munne A, Ligthart JM, Pattynama PM, Krestin GP, Serruys PW, de Feyter PJ (2002) Usefulness of multislice computed tomography for detecting obstructive coronary artery disease. Am J Cardiol 89:913-918

10. Hong C, Becker CR, Schoepf UJ, Ohnesorge B, Bruening R, Reiser MF (2002) Coronary artery calcium: absolute quantification in nonenhanced and contrast-enhanced multi-detector row CT studies. Radiology 223:474-480
11. Rubin GD, Schmidt AJ, Logan LJ, Sofilos MC (2001) Multi-detector row CT angiography of lower extremity arterial inflow and runoff: initial experience. Radiology 221:146-158

12. Flohr T, Stierstorfer K, Bruder H, Simon J, Schaller S (2002) New technical developments in multislice CT. I. Approaching isotropic resolution with sub-mm 16-slice scanning. Rofo Fortschr Geb Rontgenstr Neuen Bildgeb Verfahr 174:839-845

13. Flohr TG, Schaller S, Stierstorfer K, Bruder H, Ohnesorge BM, Schoepf UJ (2005) Multi-detector row CT systems and image-reconstruction techniques. Radiology 235:756-773

14. Brix G, Nagel HD, Stamm G, Veit R, Lechel U, Griebel J, Galanski M (2003) Radiation exposure in multi-slice versus single-slice spiral CT: results of a nationwide survey. Eur Radiol 13:1979-1991

15. Yates SJ, Pike LC, Goldstone KE (2004) Effect of multislice scanners on patient dose from routine $\mathrm{CT}$ examinations in East Anglia. Br J Radiol 77:472-478

16. Hart D, Wall BF (2004) UK population dose from medical X-ray examinations. Eur J Radiol 50:285-291

17. Shrimpton PC, Hillier MC, Lewis MA Dunn M (2005) Dose from computed tomography examinations in the UK-2003 Review NRPB Report W67 Chilton: National Radiological Protection Board

18. Aroua A, Vader JP, Valley JF and Verdun FR Exposure of the Swiss population by radiodiagnostics: 2003 Review Health Physics (submitted 2005)

19. International Electrotechnical Committee 1994. Medical diagnostic $\mathrm{X}$-ray equipment-Radiation conditions for use in the determination of characteristics. Standard IEC \# 61267, Geneva Switzerland

20. Verdun FR, Noel A, Meuli R, Pachoud M, Monnin P, Valley JF, Schnyder P, Denys A (2004) Influence of detector collimation on SNR in four different MDCT scanners using a reconstructed slice thickness of $5 \mathrm{~mm}$. Eur Radiol 14:1866-1872
21. http://www.impactscan.org

22. European guidelines on quality criteria for computed tomography 1999: report EUR 16262. Available at: http://www. $\mathrm{drs} . \mathrm{dk} /$ guidelines/ct/quality/mainindex. htm

23. Federle MP (2005) CT of the acute (emergency) adbdomen. Eur Radiol 15 (Suppl 4):D100-D1004

24. Leschka S, Alkadhi H, Wildermuth S, Marincek B (2005) Multi-detector computed tomography of acute abdomen. Eur Radiol 15:2435-2447

25. Farber JM, Buckwalter KA (2002) Sports-related injuries of the shoulder: instability. Radiol Clin North Am 40:235-249 Mar

26. Bitzer M, Nasko M, Krackhardt T, Schick F, Schober W, Wiskirchen J, Morgalla M, Weise K, Claussen C (2004) Direct CT-arthrography versus direct MR-arthrography in chronic shoulder instability: comparison of modalities after the introduction of multidetector-CT technology. Rofo 176 (12): 1770-1775

27. Hesse B, Murphy R, Greenberg N, Gring C, Sauri D, Garcia M (2005) Tissue Doppler imaging-guided prospective tube current modulation in cardiac multidetector computed tomography. Abstract SSK08-04, 91st annual meeting of the Radiological Society of North America, Chicago 2005

28. Gurung J, Khan MF, Maataoui A, Herzog C, Bux R, Bratzke H, Ackermann H, Vogl TJ (2005) Multislice CT of the pelvis: dose reduction with regard to image quality using 16-row CT. Eur Radiol 15:1898-1905

29. Greess H, Lutze J, Nomayr A, Wolf H, Hothorn T, Kalender WA, Bautz W (2004) Dose reduction in subsecond multislice spiral CT examination of children by online tube current modulation. Eur Radiol 14:995-999 